\title{
Norois
}

Environnement, aménagement, société

\section{Questions sur la neutralité des outils de type jeux de rôle et cartographie participative dans une expérience de gouvernance foncière au Sénégal}

Questions on the Neutrality of Participative Cartography and Role Play Tools into a Land Tenure Governance Experience in Senegal

Laurence Boutinot, Alain A. Viau et Grégoire Leclerc

\section{(2) OpenEdition}

Journals

\section{Édition électronique}

URL : https://journals.openedition.org/norois/2641

DOI : $10.4000 /$ norois. 2641

ISBN : 978-2-7535-1557-4

ISSN : $1760-8546$

Éditeur

Presses universitaires de Rennes

\section{Édition imprimée}

Date de publication : 15 décembre 2008

Pagination : 73-89

ISBN : 978-2-7535-0805-7

ISSN : 0029-182X

Référence électronique

Laurence Boutinot, Alain A. Viau et Grégoire Leclerc, «Questions sur la neutralité des outils de type jeux de rôle et cartographie participative dans une expérience de gouvernance foncière au Sénégal », Norois [En ligne], 209 | 2008/4, mis en ligne le 01 mars 2011, consulté le 14 janvier 2022. URL : http:// journals.openedition.org/norois/2641; DOI : https://doi.org/10.4000/norois.2641 


\title{
Questions Sur LA NEUTRALiTÉ Des OUTILS DE TYPE JEUX DE RÔLE \\ ET CARTOGRAPHIE PARTICIPATIVE DANS UNE EXPÉRIENCE DE GOUVERNANCE FONCIÈRE AU SÉNÉGAL
}

\author{
LAURENCE BOUTINOT \\ CIRAD \\ (Université Laval), \\ Pavillon Casault, Local 3731 - G1K 7P4 QuéBEC, Qc, Canada \\ laurence.boutinot@cirad.fr,Alain.viau@scg.ulaval.ca \\ Alain A. ViaU \\ (Université Laval), \\ Faculté de foresterie et de géomatique \\ Pavillon Casault, Local 3731 - G1K 7P4 QuéBEC, Qc, Canada \\ alain.viau@scg.ulaval.ca \\ GRÉGOIRE LECLERC \\ CIRAD \\ LERG, École Supérieure Polytechnique \\ (Université Cheikh Anta Diop), \\ BP 25275, Dakar Fann, Sénégal \\ gregoireleclerc@cirad.fr
}

\section{RÉSUMÉ}

Les processus de décentralisation engagés dans un grand nombre de pays africains permettent le transfert d'importantes compétences depuis l'Etat vers les collectivités locales, et notamment, pour ce qui concerne le Sénégal, en matière de gestion des ressources naturelles et du foncier. De tels contextes politiques et institutionnels privilégient une participation élargie des populations aux décisions. Des outils participatifs tels que développés par les Méthodes d'Apprentissage Rapide et de Planification $\left(M A R P^{l}\right.$ ) se présentent comme des supports privilégiés d'aide à la concertation et aux négociations locales entre des acteurs aux intérêts et aux statuts différents. Loin d'être neutres dans leur usage, ces outils ont cependant partie liée avec une certaine neutralité en raison du caractère immanent de cette dernière et de la garantie de scientificité qu'elle produit. Dans le même temps, ils participent, en tant que supports de représentation, d'informations et de communication, au glissement des signes conventionnels vers des nouvelles variables concrètes jugées utilisables par les acteurs dans leurs négociations. Cet article propose un regard extérieur sur une expérience réalisée au Sénégal à partir d'outils participatifs, tels les jeux de rôle et l'apprentissage à la cartographie, dans un contexte de gestion foncière. L'analyse veut ques- 
tionner ces outils qui tentent de reproduire une certaine forme de réalité. Les démarches expérimentales ouvrent la voie à des questions éthiques, méthodologiques et scientifiques. Autour de ces questions, cet article contribue à évaluer le potentiel opérationnel de ces méthodes et veut en même temps rendre compte de la façon dont celles-ci limitent notre capacité à prendre en considération la réalité des rapports sociaux dans des approches de gouvernance participative. Par ce regard critique sur un cadre expérimental se donnent à voir des formes d'objectivation des pratiques des acteurs et des chercheurs; ces derniers étant de plus en plus confrontés à des situations de recherche-action. L'analyse permet de mieux évaluer l'efficacité et la portée des outils utilisés dans une démarche d'accompagnement des populations dans les contextes de gestion foncière et de gouvernance locale des territoires. Les auteurs sont, respectivement, participant et observateur, associé à l'analyse et responsable du projet. Actuellement membres de la Chaire Internationale de Recherche sur la gouvernance des territoires (CIRGT) dans une collaboration du Cirad avec l'Université Laval à Québec, les deux premiers auteurs ont été associés au troisième, promoteur du projet à l'étude, pour partager une réflexion sur les enjeux que soulève l'utilisation de certains outils participatifs tels les jeux de rôle et la cartographie.

MotS CLÉ : jeux de rôle - cartographie - gouvernance - prise de décision - foncier - démarches participatives - gestion des ressources naturelles - outils - décentralisation

\section{ABSTRACT \\ Questions on the Neutrality of Participative Cartography and Role Play Tools into a Land Tenure Governance Experience in Senegal}

Decentralization movement engaged in a great number of African countries allow the transfer of important competences from the State towards the local communities, and in particular, concerning Senegal, as regards to natural resources management and land tenure. In such political and institutional contexts which privilege a widened participation of the populations in the decisions, participative tools as developed for the MARP are presented in the form of privileged supports of assistance at the dialogue and the local negotiations between actors with different interests and statutes. Far from being neutral in their use, these tools however have dependent part with a certain neutrality because of their immanent character and the guarantee of scientificity which it produces. In the same time, they take part, as supports of representation, information and communication, with the slip of the conventional signs towards new concrete variables considered to be usable by the actors in their negotiation. This article proposes an external view on an experiment carried out in Senegal starting from the use of participative tools, the such Role Play and cartography training, in a context of land tenure issues. The analysis wants to question these tools which try to reproduce a certain form of reality. The experimental steps open the way with questions of an ethical, methodological and scientifical nature. Around these questions, this article contributes to evaluate the operational potential of these methods and wants at the same time to take into account the way in which those methods limit our capacity to take into account the reality of the social issues into participative governance approaches. By this critical overview on an experimental framework are given to see different forms of objectivation of the actors and researchers practices being confronted more and more with research-action situations. The analysis makes it possible to better evaluate the effectiveness and the potential of the tools used in their accompaniment of the populations in the contexts of land tenure and local territorial governance.

KEY WORDS : role play - cartography - governance - decision making - land tenure - participation - natural resources management - tools - decentralization 
Les processus de décentralisation engagés dans la plupart des pays en développement, à l'instar des sociétés occidentales nous donnent à voir, d'une manière nouvelle et sans doute plus complexe, les problématiques de gouvernance et de gestion des espaces et des ressources naturelles qui les composent.

Le terme " gouvernance », dans son acception contemporaine, apparaît d'abord avec R.H. Coase (1988, [1937]) dans le monde de l'entreprise. Il désigne alors les différents modes de coordination et de partenariat mis en œuvre au sein d'une firme (Healey, 2005; Hufty, 2007; Viau et Boutinot, 2007; Roy et Viau, 2007). À partir des années 1980, la notion de gouvernance est élargie à d'autres domaines (Froger, 2007; Ervine, 2007; Milani, 1999). Elle est importée d'une part, des sciences politiques anglo-saxonnes afin de nourrir la réflexion sur les recompositions du pouvoir local et, d'autre part, dans le champ des relations internationales par les institutions financières qui cherchent à définir les critères d'une bonne administration publique dans les pays soumis à des programmes d'ajustement structurel (Diop 2002, 2004). Autour de l'idée de gouvernance gravitent alors des notions plus normatives de «bonne » gouvernance, de transparence dans les décisions et de démocratie qui la font alors apparaître comme la meilleure réponse possible aux problématiques de développement politique et social, en tant que nouvelle forme de régulation pour répondre à un contexte de crise (Hufty, 2007). Ce concept de gouvernance met aussi l'accent sur la transformation des modalités de l'action publique (Muller, 1990; Lascoumes et Galès, 2004 ; Duran, 2004) associant une diversité d'acteurs susceptibles d'intervenir dans la gestion des affaires publiques au-delà de l'État, telles les collectivités territoriales, mais aussi les organismes nationaux et internationaux, les différentes régions, les ONG, les entreprises privées et les citoyens.

Dans le contexte des économies mondialisées et de la prise en considération sans précédent des questions de préservation de l'environnement, les politiques territoriales et sectorielles se conjuguent tant bien que mal dans une redistribution des pouvoirs entre les autorités et des ayants droit quant à l'accès, l'usage et l'exploitation de ces ressources. Dans leur valeur ajoutée, ou simplement en tant que mode de subsistance, ces espaces et ces ressources posent à la fois le problème de leur régénération, de leur renouvellement et de leur équitable distribution eu égards aux conditions d'existence précaires des sociétés du monde rural. Ce contexte de changement favorise l'émergence de nouvelles formes de gouvernance avec la participation des populations locales au développement durable de leur territoire selon des règles du jeu souvent définies en dehors de ce dernier. Les populations ont toujours d'une manière ou d'une autre participé au processus de prise de décision en matière de gestion de leur territoire et des ressources naturelles qui y sont associées (Ayeva, 2003; Fairhead et Leach, 2003, 1998; Agrawal, 2005). L'évolution des politiques publiques liée à une redistribution des pouvoirs entre les autorités et des ayants droit par la décentralisation implique de considérer de nouvelles approches d'accompagnement afin que la participation des populations au développement soit valorisée; H. Blair (2000) en a bien fait état. En effet, la participation est théoriquement une condition de la démocratie en tant que forme propice à l'engagement des hommes dans la gestion et la décision des affaires locales et nationales. Elle représente la possibilité pour chacun de s'exprimer et d'exercer ses droits et devoirs pour la construction d'une vision citoyenne du développement (Blondiaux et Levêque, 1999) et contribue à la transformation de nos pratiques politiques (Blondiaux, 2007). Elle est également porteuse d'un potentiel d'égalité entre les hommes. Dans le cadre des opérations de développement, la participation peut être entendue comme une implication des villageois susceptible de leur octroyer une certaine autonomie d'action afin de s'approprier, à terme, les objectifs d'un projet. Mais la participation reste toutefois bien mal définie en pratique du fait des contextes divers dans lesquels elle s'exerce. Elle a pu, en outre, revêtir des formes différentes selon les époques et n'avoir pas toujours été en harmonie avec les principes qui la portent. Par ailleurs, elle peut également prendre des formes ambiguës (Boutinot et Diouf, 2006) selon les modalités de sa mise en œuvre. Aussi, dans un climat d'incertitude et de vulnérabilité face à la gestion et à l'accès aux ressources agro-pastorales et forestières des territoires, on observe, dans les sociétés rurales africaines, un regain d'intérêt pour des modes de sécurisation à travers des demandes de formalisation juridiques et contractuelles, telles les chartes locales et les codes de gestion (Djire, 
2004 ; Granier, 2006). Ces démarches requièrent cependant la participation des villageois supposée garantir la légitimité des accords.

C'est dans ce contexte que de nombreuses méthodes, outils et approches sont mobilisés pour aider les acteurs à prendre des décisions concernant les affaires publiques locales en Afrique (diagnostics participatifs, focus group, etc.). Cet article se limitera à l'analyse de deux de ces approches. La première approche est basée sur un jeu de rôle et la seconde sur l'apprentissage à la cartographie. Ces démarches ont été mises en œuvre par un projet de recherche-action au nord du Sénégal. Nous tenterons de rendre compte à la fois de la portée de ces outils en tant qu'objets intermédiaires entre les acteurs dans la démarche de co-construction d'outils de gouvernance, et de la dynamique des rapports sociaux engendrée par ces expériences.

\section{Contexte d'usage des outils participatifs pour la gouvernance et la gestion des ressources naturelles (jeux de rôle et apprentissage à la cartographie)}

La gestion des ressources naturelles relève depuis l'époque coloniale de la responsabilité des États et a souvent donné lieu à l'élaboration d'inventaires et de représentations de celles-ci sur le territoire afin de mieux orienter les stratégies de gestion. Dans le cadre de la gestion décentralisée des ressources naturelles et des territoires, les enjeux d'échelle sont reconsidérés pour répondre aux nouveaux dispositifs de gouvernance (Viau et Boutinot, 2007; Adger et al., 2003).

\section{INTÉRÊTS ET LIMITES DES OUTILS PARTICIPATIFS}

Au premier rang des outils utilisés et connus en Afrique pour l'élaboration des plans ou des chartes locales de gestion et en amont de la mise en œuvre de la plupart de projets extérieurs, on trouve la démarche participative par l'usage des Méthodes d'Apprentissage Rapide et de Planification (MARP). Ces méthodes essentiellement qualitatives ont remis en cause celles qui dépendaient de techniques plus quantitatives dans lesquelles les procédés d'échantillonnages classiques «statistiquement représentatifs » étaient accusés de masquer les diversités et les processus. Elles reprochaient également aux enquêtes lourdes et coûteuses, basées sur de longs questionnaires, de n'apporter que des traitements différés dans le temps avec des résultats considérés obsolètes avant leur utilisation. Le fait que les MARP puissent être des approches plus opérationnelles et surtout plus rapides que les méthodes classiques pour extraire des demandes sociales s'est peu à peu affirmé dans la mise en œuvre des projets de développement, auprès des ONGs mais également auprès des services publics (Boutinot, 2002). Depuis leur introduction en Afrique au début des années 1980, les MARP n'ont eu de cesse d'être pratiquées dans les contextes des projets de développement mis en œuvre par les ONGs en général et quels que soient les domaines (santé, agriculture, éducation etc) ciblés. Elles ont cependant pu faire depuis l'objet de critiques sur les plans méthodologiques (CERCOOP, 2003) et théoriques (Mosse, 2000; Lavigne-Delville et al., 2000). D'aucuns pourraient ajouter les appréhensions grandissantes des populations rurales envers les agents de développement qui pourraient contribuer à rendre difficile, dans l'avenir, le déroulement de la démarche MARP.

Pourtant aujourd'hui, la participation est devenue le libellé des projets qui ont mis en oeuvre ces types de diagnostics rapides. Mais pour rappeler les termes de P. Lavigne-Delville et al. (2000), ces approches participatives sont, d'une manière générale, ambiguës et leurs animateurs assument difficilement leur situation d'intervenants extérieurs au monde villageois. En effet ils tendent à laisser aux villageois la liberté de choisir leurs représentants dans un souci démocratique; mais, dans le même temps, les activités et les objectifs de développement qu'ils portent font, eux, l'objet d'un encadrement et de pratiques plus dirigistes. Cette ambiguïté constitue un biais principal dans la démarche qui invite à s’interroger sur la représentativité des acteurs. Les présupposés des méthodes MARP épousent cette contradiction de telle manière qu'ils permettent le plus souvent d'imposer les objectifs des projets plutôt 
que de rendre compte d'une demande sociale. Au vrai, les divers dysfonctionnements des enquêtes MARP sont intrinsèques à la méthode (Sellamna, 2000; Lavigne-Delville et al., 2000).

Si les MARP ont été présentées comme une innovation majeure dans les méthodes d'investigation utiles au monde du développement, ce n'était pas seulement dans la nécessité de substituer aux coûteuses enquêtes quantitatives, des enquêtes légères et qualitatives. Dans les sciences sociales, comme nous le rappellent P. Lavigne-Delville et al. (2000), les enquêtes qualitatives et quantitatives ne s'excluent pas et peuvent être complémentaires et, sont, de toute façon, utilisées en cohérence avec les hypothèses et l'objet de recherche à traiter. En définitive, s'il y a une innovation à l'utilisation de ces méthodes qualitatives rapides, c'est dans le fait que de plus en plus de spécialistes des sciences de la nature les intègrent dans leurs pratiques. Mais si l'appropriation de l'approche qualitative, propre aux sciences sociales, par des spécialistes pas nécessairement formés à sa mise en œuvre est sans doute l'un des apports majeurs des démarches participative (Lavigne-Delville et al., 2000), il reste que « le risque de dérapage est grand, dès lors que les conditions d'enquête ne sont pas maîtrisées » (LavigneDelville et al., 2000). Conscients de ces possibles dérives, les concepteurs des MARP ont procédé à de nombreux ajustements dans des manuels et y ont intégré des garde-fous, tels que la nécessité de réaliser des observations directes sur le contexte étudié, de prévoir des réunions d'échange au sein de l'équipe de recherche pour produire un retour réflexif sur le travail réalisé et permettre d'affiner les outils utilisés ou d'en développer d'autres, de rendre compte auprès des populations enquêtées d'une première synthèse pour produire un retour d'information et partager les commentaires, etc. (Gueye et Tall, 2001 ; Rambaldi et al., 2006). Il reste cependant que certains éléments fondamentaux révèlent la limite et la rigidité propres à ce genre d'outil clé en main. Car cela va bien au-delà. Il est connu des chercheurs en sciences sociales qu'il n'existe pas de véritable « recette » qui permette de faire l'économie d'une longue pratique du terrain, seule productrice d'un savoir-faire et d'un savoir-être dans la relation d'enquête. Cette distance et cette réflexivité que le chercheur en sciences sociales intériorise au fil de ses travaux de terrain sont non seulement l'objet d'un temps long de l'apprentissage du métier, mais également la pierre d'achoppement qui ne fait des MARP qu'une trousse à outils bien souvent fort mal utilisée.

\section{CONTEXTE DE L’EXPÉRIENCE ET DÉROULEMENT DES ATELIERS}

En définitive les expériences sont si nombreuses et si variées qu’elles rendent malaisée la comparaison et interdisent les conclusions hâtives. De fait, certains projets participatifs tentent de dépasser les MARP, d'une part en intégrant l'observation participante au dispositif, d'autre part en laissant de côté la boite à outils MARP pour développer, et si possible co-construire, de nouveaux outils qui soient mieux adaptés aux enjeux et aux personnes qui les portent. Même si les nouveaux outils peuvent être relativement normés (ou pré-construits), la posture des chercheurs est de permettre aux acteurs de les remettre en question, en considérant leur usage dans les prises de décision. C'est ce type de démarche que nous interrogeons en nous inspirant d'une expérience qui s'est déroulée autour du Lac de Guiers situé au nord du Sénégal (fig. 1), relative à la gouvernance des territoires et des ressources naturelles (Leclerc et al., 2008). Cette expérience avait pour objectif à terme de co-construire, avec les acteurs, décideurs et usagers, des outils de modélisation pour venir en appui aux modes de gouvernance en matière de gestion des terres. Le territoire concerné par cette expérience couvre une superficie de $10000 \mathrm{~km}^{2}$ et implique des acteurs issus de divers niveaux d'organisation et de responsabilités. Ce territoire comprend la ville de Richard-Toll, le lac de Guiers - qui couvre 30 \% des besoins en eau potable de Dakar - le fleuve Sénégal et les terres frontalières avec la Mauritanie toute proche (fig. 1). La problématique foncière dans ce contexte reste étroitement liée à des activités diversifiées au sein d'un même espace : agriculture, pêche, tourisme cynégétique et production pastorale. Ce projet couvre plusieurs communautés rurales ${ }^{2}$

2. Une communauté rurale est la collectivité territoriale la plus décentralisée. Elle comprend entre 50 et 200 villages selon les régions. Elle est administrée par un Conseil rural élu au suffrage universel depuis la loi de la décentralisation de 1996. Dans un souci d'anonymat, les noms des communautés rurales concernées dans cet article ne sont pas mentionnés. 
et touche trois arrondissements, deux départements et deux régions et doit composer avec une échelle différente des unités habituellement mobilisées dans les projets de gestion des ressources naturelles de la région, tel le terroir (village) ou l'unité pastorale ${ }^{3}$.

Les premières démarches du projet ont consisté en une réunion d'information et de présentation des objectifs dans les communautés rurales concernées. Cette première étape a donné lieu à la formalisation d'un « groupe de travail » composé, outre des 5 chercheurs, de 17 représentants locaux à la fois pressentis et volontaires parmi les participants présents. Ces derniers provenaient, du groupe des élus locaux, du monde associatif, des organisations de producteurs, des autorités traditionnelles (chef de village, chef religieux), des sociétés de développement et de la population ordinaire.

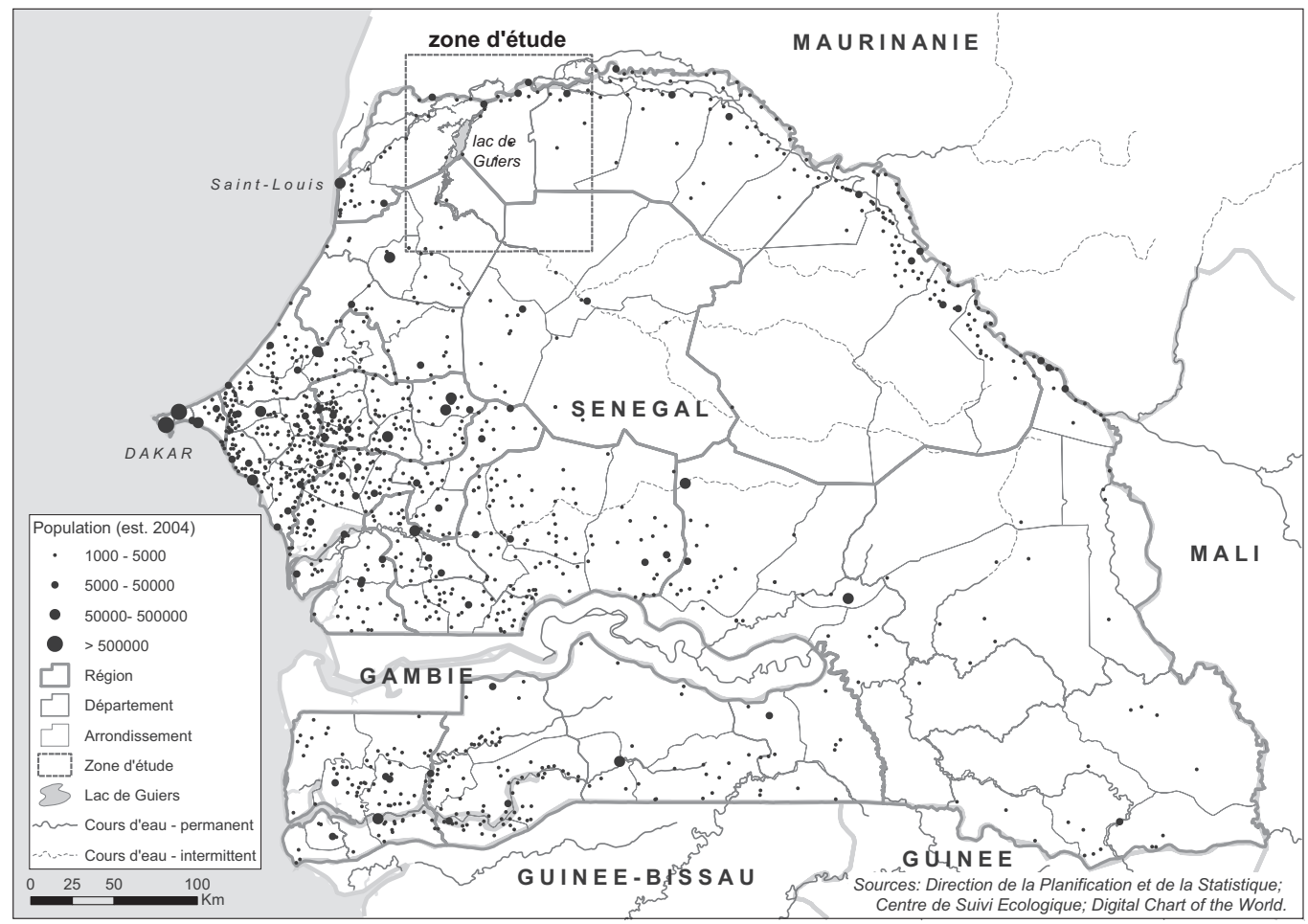

Figure 1 : Région du Lac de Guiers au nord du Sénégal Lake of Guiers area, north of Senegal

Plusieurs ateliers d'une durée de deux à trois jours ont ensuite été réalisés avec ce groupe de travail dans le cadre du processus participatif initié. Les deux premiers ont mobilisé deux types d'outils, soit le jeu de rôles et la formation à la cartographie, qui sont analysés pour les fins du présent article.

Le premier atelier a été réalisé en octobre 2006 dans la salle de réunion du conseil rural de Keur Momar Sarr. Il a débuté avec un rappel des objectifs du projet et la présentation de soi. Les chercheurs avaient essentiellement un rôle d'animation et d'observation. La méthode d'animation

3. Une unité pastorale est une forme plus ou moins institutionnalisée de regroupement de familles d'éleveurs transhumants et d'agro-éleveurs sédentaires autour d'un forage et de son aire de desserte, ou rayon de pâturage (UBT/ha) et qui forme un comité ad hoc pour la gestion et l'entretien du forage. Ces unités pastorales ont été mises en place, malgré la décentralisation, par le projet d'appui à l'élevage (PAPEL) du ministère de l'Agriculture et de l'Élevage au Sénégal. 
employée a ensuite consisté en un jeu de rôle dont les règles minimalistes ont été exposées : quelques participants, représentant des groupes sociaux (femmes) ou professionnels (éleveurs, maraîchers) acceptant de jouer le jeu, ont été sollicités pour concevoir un projet de demande foncière et le présenter au groupe. Chacun de ces «acteurs », au sens théâtral du terme, était relativement familier avec le discours public, soit en tant que « courtier en développement » (Bierschenk et al., 2000), soit en tant que personne publique locale. Les chercheurs ont ensuite fait un bref retour de l'expérience dans le groupe, discutant les chances réelles de recevabilité des demandes dans les délibérations d'un conseil rural. Le second atelier, dont l'objectif était de renforcer les capacités des membres du groupe de travail en matière de cartographie, a été réalisé à Richard-Toll en avril 2007, dans la salle de réunion d'une ONG partenaire (le Centre de gestion et d'économie rurale du Walo). De manière pédagogique, les objectifs du projet et les résultats de l'atelier précédent ont été rappelés en début de séance où le besoin d'aider les acteurs locaux à acquérir une culture cartographique critique (Johnson et al, 2006) avait été identifié. Cet atelier était la première étape d'un processus d'apprentissage visant à permettre aux acteurs de lire des cartes (de maîtriser le symbolisme cartographique, la notion d'échelle), d'utiliser (et de concevoir) des cartes dans la prise de décision (D’Aquino, 2001). L'animation a consisté en un enseignement assez classique d'explication des éléments de base de la cartographie (titre, orientation, légende, échelle) et qui était composé d'exercices «au tableau » sur des cartes de complexité croissante. La première carte représentait les unités morpho-pédologiques et les cours d'eau; à la seconde, s'ajoutaient les infrastructures hydrauliques; à la troisième, l'habitat et les routes. Les participants ont été amenés à voir l'adéquation entre les dessins et les légendes, à situer un point sur la carte et le dessiner, à localiser les villages les uns par rapport aux autres, enfin à mesurer la distance entre deux points en utilisant l'échelle, voire à corriger les erreurs d'une carte jugée trop ancienne ou à proposer de nouvelles informations nécessaires pour la prise de décision.

\section{Le jeu de rôle : Intérêt et risque de l'euphémisation}

\section{LES OBJECTIFS DU JEU DE RÔLE}

L'objectif affiché par le premier atelier était de susciter un débat sur la terre de la région à travers l'expérimentation d'une situation fictive de demande foncière. Dans cette simulation, les acteurs ${ }^{4}$ devaient improviser une demande de terre pour un projet agricole et l'argumenter devant le groupe de travail censé représenter un conseil rural en séance de délibération. En soutenant leur propre projet devant une assemblée censée représenter un conseil rural, la simulation permettait, d'une part, d'analyser le type d'argumentaire utilisé par les protagonistes pour appuyer leur demande, les mécanismes et astuces pour capter l'intérêt du groupe de travail et, d'autre part, d'observer les enjeux virtuels que soulève une demande foncière. Si le jeu de rôle avait pour ambition implicite de rendre compte des relations entre les protagonistes, il avait également pour objectif d'inviter les membres du groupe de travail à se constituer en collectif dans un lieu d'échange et de communication autour des questions foncières. Quand bien même les statuts respectifs des membres du Conseil Rural et de ceux du groupe de travail n'étaient pas de même nature dans la réalité, le contexte du jeu a pu les confondre parfois dans l'esprit des participants. Ceux-ci pouvaient en effet sortir de leur statut pour « faire comme si » leur parole équivalait celle d'autrui dans le règlement des questions foncières. Cette confusion présentera, sans nul doute, une limite forte du passage de la simulation à la réalité.

Le premier intérêt du jeu de rôle se situe dans l'efficacité qu'il produit quant à la dynamique des échanges entre les acteurs (chercheurs et populations villageoises confondus). Cependant, si les échanges ont été pertinents, ils ont aussi donné à voir la capacité des acteurs à jouer en toute

4. Trois «acteurs » ont été identifiés. Le « choix» des acteurs a permis d’aborder les trois principaux types de problèmes rencontrés dans la gestion foncière (terres maraîchères, territoire pastoral, équité dans le genre). 
«bonne foi ${ }^{5}$ ». Autrement dit, les acteurs ont simulé une situation sans toutefois jamais rien lâcher de leur rapport au réel et sans désolidariser leur jeu de leurs pratiques réelles au quotidien, i.e. leurs revendications sur le foncier, qui, à l'occasion, pouvaient prendre réalité. À travers le jeu se dessine l'hypothèse selon laquelle le recul des acteurs sur leur situation est susceptible d'ouvrir à des possibilités de mobilisation et de projection dans un futur proche et possible. Pourtant le jeu des acteurs n’a pas évacué les rapports sociaux réels, les enjeux ni les rapports de domination qui peuvent se lire à travers cette expérience.

\section{INTÉRÊT ET RISQUES DE L’EUPHÉMISATION}

C'est aussi parce qu'il existait des liens étroits entre les acteurs et dans leur rapport à leur réalité, que cette simulation a révélé certaines stratégies politiques et de requêtes de leur part, transformant le cadre fictif en une réalité. Par exemple, la demande foncière pour un groupement de femmes se donnait à voir comme une transposition d'un fait réel qui n'avait pas bénéficié de la faveur des décideurs. Il s'est avéré, dans cet exemple, que les acteurs ont utilisé cette simulation comme une seconde chance pour présenter leur requête. De la sorte, les acteurs ont pu souligner publiquement, dans leur argumentaire, certaines pratiques indicibles de la part des élus qui constituent le leitmotiv des revendications des villageois dans la distribution foncière.

L'intérêt du jeu de rôle est-il alors dans ce pouvoir métonymique de rendre compte d'une réalité sociale à partir de l'évocation d'un seul exemple? L'intérêt du jeu de rôle se trouve-t-il aussi dans ce pouvoir d'évocation d'une situation mi fictive, mi réelle, où toute la dimension ambiguë permet aux rapports de force locaux de s'exprimer de façon plus ou moins euphémique, du fait de son caractère public et ludique en même temps? Les jeux de rôle ont, par ailleurs, dans le choix des acteurs et de leurs histoires, donné à voir l'importance différentielle des modes de négociation utilisés selon les cas de figure; nous rappelant, si besoin était, que le jeu pouvait faire ressortir des registres argumentaires bien réels. En effet, les trois cas de figure choisis (courtier, femmes, éleveurs) ont présenté des registres de négociations différents, selon la nature des demandes et le statut des demandeurs. Cette simulation a permis de révéler cinq types d'argumentaires récurrents dans les demandes foncières (plus d'un argument peut se retrouver dans une même demande).

Le premier type d'argumentaire, que nous pourrions appeler " politiquement correct », relève d'un registre idéologique représenté dans les discours de la politique nationale et internationale du Sénégal ${ }^{6}$. La présence des chercheurs occidentaux dont une femme, dans le dispositif a pu favoriser les déclarations d'intention en faveur de l'égalité de genre. Ainsi, la demande foncière présentée par une femme, par ailleurs chargée des questions de genre et de développement agricole dans un média local, a pu avoir un impact plus réel que fictif dans le dispositif mis en place. Cette demande foncière, présentée au nom d'un groupement de cent femmes productrices maraîchères dans le cadre du jeu de rôle, a pu contraindre quelque peu les décideurs présents dans le groupe de travail d'accéder publiquement à sa requête. Ces derniers ont dû ainsi avancer publiquement l'argument selon lequel un élu «peut bloquer la demande d'un homme mais pas d'une femme » et qu'il «fera tout pour aider une femme ».

Le deuxième type d'argument, que nous intitulons « clientélisme et légalité », relève du registre délicat de la «petite corruption ». Bien que celle-ci soit sous une forme «banalisée et généralisée $[. .$.$] et même si grande et petite corruption forment à l'évidence un continuum » (Blundo et$ Olivier de Sardan, 2001), elle a pu être présentée comme un élément dominant les transactions foncières. En effet, une des demandes présentées dans le jeu a évoqué la nécessité d'une pratique corruptive pour accéder aux faveurs des décideurs et a permis de révéler à la fois la régularité

5. En référence à la pièce de théâtre de Marivaux, Les acteurs de bonne foi (1757). Mais, du même auteur, on pourrait également citer L'̂̀le des esclaves (1725).

6. Le Président Abdoulaye Wade a proposé l'adoption de la politique de parité homme/femme dans la Commission de l’Union africaine en 2002. 
de ce type de pratique et l'indignation des acteurs. « Le foncier demeure (donc) un des rares domaines où les conseillers ruraux gardent un contrôle et conservent par là leur propre réseau de clientèle » (Blundo, 1997). De plus, « dans la région du Fleuve, les conseils ruraux sont contrôlés par les couches sociales d'origine noble et les décisions foncières respectent la hiérarchie sociale » (Blundo, 1997).

Un troisième type d'argumentaire avait trait à la protection de l'environnement. Ici, la présentation de la demande foncière concernait la réalisation d'un projet de reboisement, le long du lac de Guiers, afin de former une zone tampon susceptible de protéger le lac de la pollution engendrée par les productions de patates douces sur les berges. Cette demande mettait en relief et interpellait les intérêts bien compris pour la modernisation agricole, les études d'impact et la valeur ajoutée des projets soucieux de l'environnement.

Un quatrième type d'argument concernait le rapport autochtone/allochtone et avait un caractère générique. En effet, quels qu'aient été les objectifs des différentes demandes foncières présentées, chacune d'entre elles a fait l'objet de débats sur l'origine des ayants droit et sur leur statut résidentiel dans la communauté rurale. Depuis la loi sur le domaine national de 1964 et malgré le processus récent de réforme de la Loi d'Orientation Agro-sylvo-pastorale (LOASP), le rapport entre le droit coutumier et les prérogatives attribuées aux conseils ruraux n'est pas clairement statué et donne lieu à des conflits, souvent inextricables, entre les différents statuts des acteurs (natifs, résidents, autochtones, allochtones, immigrés, voire réfugiés ${ }^{7}$ ), qui attisent les jeux de pouvoir et le factionnalisme politique (Blundo, 1997).

Enfin, le dernier registre argumentaire concernait la place des éleveurs et l'on peut également le considérer comme un phénomène récurrent dans les conflits potentiels relatifs à la gestion foncière. La demande foncière présentée par le représentant des éleveurs, bien qu'elle ait été argumentée sur un registre modernisateur, a ouvert le débat sur les problèmes de transhumance pastorale. Du point de vue de certains acteurs « les problèmes viennent des transhumants quand ils ne demandent pas de terre, et aussi quand ils en demandent une ».

À travers ces exemples apparaissent à la fois la force et les limites du potentiel expérimental des jeux de rôles. En effet, au-delà de la communication, des supports de dialogue, ou des registres de l'argumentaire, la simulation a révélé un problème de statut mais sans le changer dans la réalité. Et malgré le caractère ludique de l'expérience, des acteurs participants ont pu rester dubitatifs sur les règlements des questions foncières concrètes, à travers les non-dits et les ambiguïtés liés au contexte du jeu. Si l'expérience du jeu de rôle permet une énonciation libre de problèmes fonciers, elle ne détient pas pour autant un pouvoir immanent pour les résoudre.

\section{L'apprentissage à la cartographie}

\section{Mise EN PLACE D'Un SUPPORT DE REPRÉSENTATION CARTOGRAPHIQUE DU TERRITOIRE POUR LE DÉBAT}

L'objet du deuxième atelier a été d'évaluer l'apport des outils cartographiques dans les démarches de prise de décision associées aux questions foncières (photo 1). L'utilisation d'une carte existante dans une démarche formative a favorisé la familiarisation et l'appropriation de l'outil cartographique par les acteurs participants. Cependant, il convient de distinguer la carte en tant que support de communication et la communication elle-même, en tant que message véhiculé d'un locuteur à un récepteur. Cette distinction est nécessaire afin de saisir ce qui relève du savoir d'expert, en tant que processus cognitif, de ce qui relève du savoir social dans l'apprentissage et ce, bien qu'on ne puisse « isoler l'objet de son usage » (Desrosières, 2000). Le savoir des acteurs en apprentissage vient en retour modifier le savoir social de la carte, en tant que support médiatique.

\footnotetext{
7. Suite au conflit sénégalo-mauritanien de 1989.
} 
À ce titre, N. Chrisman $(2005,2007)$ l'a clairement démontré, ainsi que ceux qui ont utilisé les systèmes d'information géographique participatif (Harvey, 2000; Harvey et al., 1998) et questionné la construction sociale de tels systèmes (Sieber, 2000). Mais le savoir des acteurs n'est pas non plus sans effet sur le savoir de l'expert et invite celui-ci à modifier son outil. Les notions de légende et d'échelle, par exemple, nous ramènent à des représentations de l'espace bien spécifiques et étroitement liées à des pratiques quotidiennes, que l'expression en langue vernaculaire permet de révéler. Elles doivent donc être traitées avec précaution car elles impliquent l'utilisation d'une sémiologie graphique et des modes de classification qui sont, à la fois du point de vue cognitif et du point de vue social, différentes des normes géographiques conventionnelles. En tant que support de communication et de dialogue entre les différents acteurs concernés par la gestion d'un territoire et des ressources naturelles qu'il renferme, l'approche par l'apprentissage à la cartographie avait pour objectif de favoriser une concertation des acteurs au niveau de la représentation de leur territoire tout en valorisant les dimensions perceptuelles de chacun. La recherche d'un consensus entre ces acteurs dans les décisions d'aménagement était aussi l'objectif des promoteurs de l'expérience. Pourtant si l'on n'interroge pas la notion même de consensus, celle-ci risque d'apparaître davantage comme un postulat de départ et non pas telle une construction empiriquement souhaitée et informée. Une des limites observée dans les processus d'apprentissage à la réalisation d'une carte relève de la forme parfois très scolaire en termes de pédagogie et se rend visible, bien souvent, dans la disposition des acteurs et les lieux d'apprentissage. Fréquemment situé dans un chef-lieu de communauté rurale en Afrique, le lieu de l'apprentissage est une salle de classe, garante d'une efficacité puisque l'école est le lieu de l'autorité du savoir. Bien souvent, la disposition scolaire classique révèle la forme d'un apprentissage descendant où l'élève répond au maître et où le savoir est dispensé et consommé. Les conditions pour une pédagogie itérative ne sont pas, dans ces conditions, totalement rassemblées et contraignent davantage à un apprentissage répétitif. J.-P. Ilboudo (2002) «pense que tous les outils qui utilisent des dessins (comme les cartes) sont jugés trop compliqués pour les paysans pour plusieurs raisons. Ceux-ci n'ont pas l'habitude de réaliser de telles activités et la persistance peut créer une situation de réticences irréversibles » (Yoda, 2004). De la même manière que certains supports telles les cartes ou les transects réveillent des craintes sur les questions foncières, les papiers, tableaux, couleurs, peuvent également être un frein à la communication et « peuvent créer un sentiment de mystification plus grand que celui des méthodes traditionnelles de recherche » (Mosse, 2000). Une disposition plus conviviale et interactive montre cependant que les formes d'apprentissage se créent davantage dans les échanges, c'est-à-dire, en partie, dans un processus de libération des inhibitions liées à l'effet que l'autorité du savoir produit sur des acteurs en grande partie non alphabétisée en langue française.

\section{EXTÉRIORITÉ OU INDÉPENDANCE DE L'OUTIL CARTOGRAPHIQUE COMME SAVOIR D’EXPERT}

L'extériorité ou l'indépendance de l'outil cartographique comme savoir d'expert est un élément important pour garantir à la fois les caractères de scientificité et d'efficacité; en d'autres termes, de rendre compte d'une certaine «neutralité » de la carte. Cette dimension est d'autant plus spécifique et visible qu'elle se présente dans un processus d'apprentissage. Mais ce caractère « neutre » de la carte produit également des effets d'imposition qui relèvent de la croyance dans le savoir expert, comme élément symbolique. Enfin, la carte produit des formes normatives ou normalisantes de la représentation de l'espace et ce, de manière d'autant plus implicite qu'elle relève d'un procédé qui semble autonome et intrinsèque à l'outil.

La distinction de la carte en tant qu'outil et de la carte comme message d'information est nécessaire avant tout pour interroger l'apparente neutralité de la carte, laquelle lui confère plus d'autorité. Mais la carte est-elle neutre? À ce titre plusieurs travaux ont largement discuté de cette neutralité (Monmounier, 1993). Ces derniers ont distingué la carte en tant qu'outil de la carte comme message d'information, révélant, si besoin était, que la carte n'est pas le territoire et en particulier lorsqu'il s'agit de représenter ce dernier à des échelles inadéquates pour reproduire 

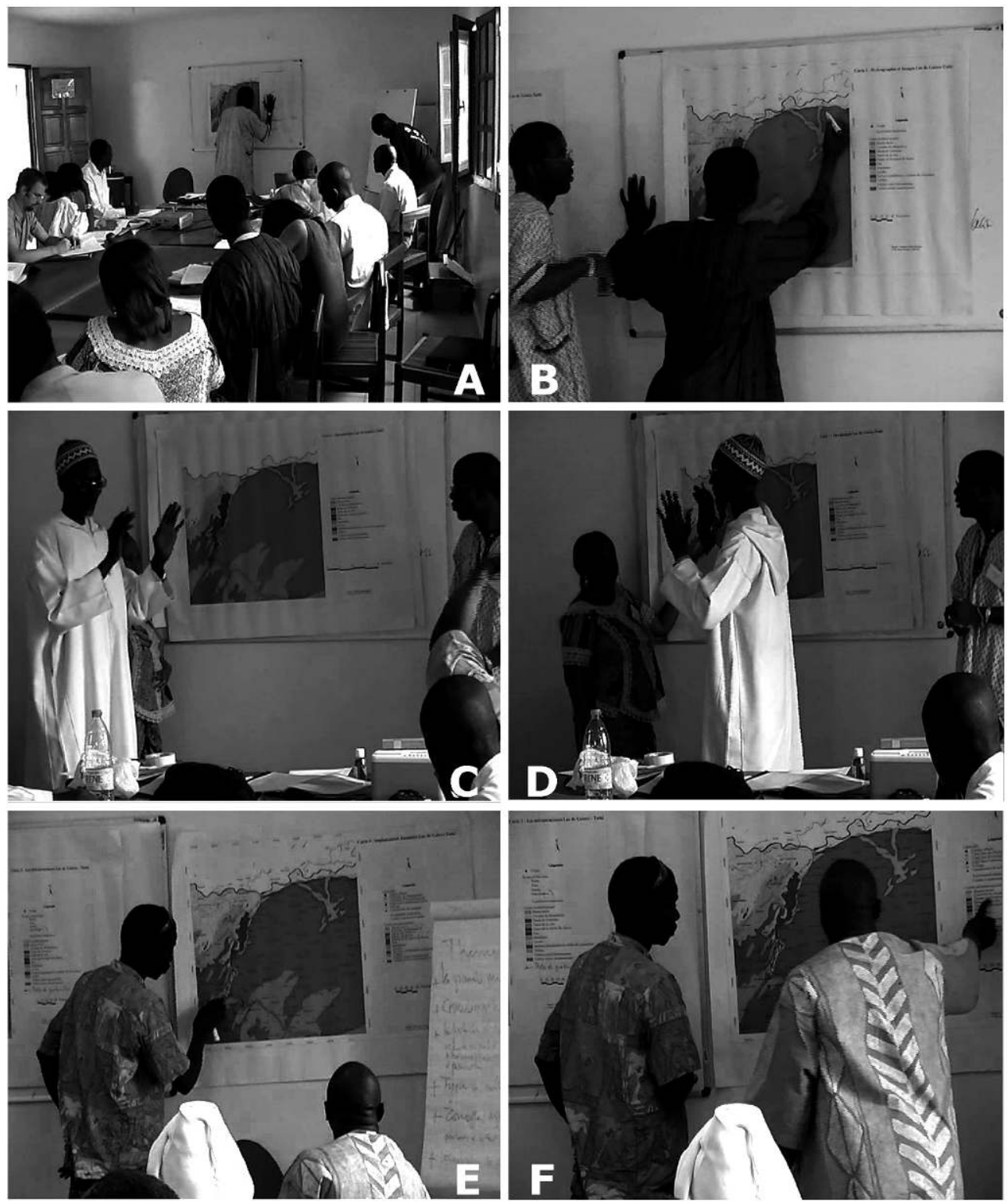

Photo 1 : Planche photographique (Leclerc, 2007) de l'atelier de formation à la cartographie

A et B : Demande de localisation d'une information jugée utile, par un éleveur (couloir de transhumance); $\mathrm{C}$ et D : Explication de la notion d'orientation est-ouest de la carte, par un Imam; E et F : Localisation par un acteur de son village assisté d'un agent technique de la SAED (Société Nationale d'Aménagement et d'Exploitation des Terres du Delta du Fleuve Sénégal).

Cartography training session pohotographies (Leclerc, 2007)

$A$ and B: Demand for localization of information considered to be useful, by a stockbreeder (transhumancy corridor); $C$ and D: Explanation by an Iman of the East-West map orientation principle; E and F: Localisation by an actor of his Village assisted by a SAED (Société Nationale d'Aménagement et d'Exploitation des Terres du Delta du Fleuve Sénégal) technical agent. 
fidèlement la réalité des terrains, la répartition des ressources naturelles, les fonctions d'usage, et tout ce qui relève du patrimoine, du bâti, et, a fortiori du culturel, du spirituel et de l'intangible. Ainsi la carte influence la prise de décision et peut exclure les utilisateurs lorsqu'elle n'est pas un portrait fidèle de la réalité du territoire perçu par ces derniers (Cambrézy et De Maximy, 1995).

En vérité, l'usage des cartes a été historiquement considéré comme un instrument de guerre (Lacoste, 1982), de commerce et de revendication territoriale avant d'être un instrument de référence scientifique ou d'usage quotidien pour représenter les territoires et les espaces géographiques avec des conventions internationalement reconnues et un cadre méthodologique de production. En cela, elle ne saurait être un simple outil d'apprentissage de l'art pour lui-même. La carte n'est pas le territoire et la représentation du territoire est à la base perceptuelle et interprétative. Par exemple, la carte utilisée, dans le cadre du présent projet, est la carte de la région du lac de Guiers, plus ou moins « vraie » ou complète, mais qui représente, de fait, une certaine réalité du terroir en question. Le jeu ambivalent de l'apprentissage à la cartographie oscille entre la réalité concrète et l'abstraction pure et ne précise pas aux participants si la carte est un simple outil, ou bien un outil fonctionnel, et dans le cas où il s'agit d'un outil fonctionnel, sa finalité concrète. Cette expérience nous montre que le caractère apparent de neutralité de la carte peut rester inscrit dans le mode d'apprentissage lui-même. Bien que celui-ci valorise la perception des participants sur les lieux réels à cartographier, il est dans le même temps contraint dans la nomenclature d'usage. La pédagogie associée à l'usage de la carte géographique consiste ainsi en un apprentissage progressif, d'une carte simple à une carte de plus en plus complexe, accompagné d'exercices (sur les légendes et sur les échelles). La neutralité de la carte est, de la sorte, préservée également dans la forme répétitive de l'apprentissage.

L'exercice a permis de faire ressortir un certain nombre de questions quant à la réalité géographique représentée sur la carte (limites administratives, emplacement des villages, des champs agricoles, des parcours de transhumance), en lien avec la dimension perceptuelle des participants. La représentation cartographique du territoire devient un enjeu dans les processus décisionnels en raison de son cadre formel qui structure autant les modes d'acquisition des connaissances que leur usage dans les prises de décision.

\section{Discussion : du pouvoir délégué aux jeux de rôle et à la carte géographique et son impact sur les rapports sociaux}

À travers ces expériences menées dans le Nord du Sénégal, il apparaît important d'élucider les formes de délégation de pouvoir (Lascoumes et Le Galès, 2004) que les acteurs octroient à certains instruments (données, jeux de rôles, modes de représentation et de visualisation du territoire par la cartographie, mode de consultation et cadre de concertation autour des savoirs et des intérêts) et qui modifient leur capacité à décider, à négocier et à mettre en œuvre les politiques publiques de gestion de l'environnement (empowerment ${ }^{8}$ pour certaines couches défavorisées ou marginalisées, rapport à l'imputabilité ${ }^{9}$ pour les acteurs élus, etc.). Ces outils d'aide à la décision produisent des effets sociaux, (tels que, par exemple, celui de mettre au même niveau de connaissance devant une carte, un Président de Conseil Rural et un éleveur), qui influencent les relations de pouvoir autour des enjeux territoriaux et de la gestion des ressources naturelles.

Lors du jeu de rôles, par ailleurs, il est intéressant de remarquer que les questions foncières n'ont pas été réellement abordées en termes concrets de localisation. Du fait de la situation ludique, les lieux ont été (notamment pour la demande de la femme et de l'éleveur) abstraitement évoqués sans emplacement (village, Communauté rurale) précis ${ }^{10}$. Les demandes des terres n'ont ainsi

8. M.-H. Bacqué (2005) définit ainsi la notion d'empowerment : « Elle indique le processus par lequel un individu ou un groupe acquiert les moyens de renforcer sa capacité d'action, de s'émanciper. Elle articule ainsi deux dimensions, celle du pouvoir, qui constitue la racine du mot, et celle du processus d'apprentissage pour y accéder». p. 32. Cf. Bibliographie.

9. Traduction du terme anglais accountability (Agrawal et Ribot, 1999).

10. Notons cependant qu'il appartient au Conseil Rural de définir la localisation exacte des terres d'une localité donnée qu'il affectera. 
pas fait l'objet d'une localisation exacte sur un dessin ou une carte, exceptée pour la demande de terre visant le reboisement d'une importante section des berges du lac, mais dont l'argumentaire a été illustré par un croquis approximatif. Ces remarques sont directement liées à la question de l'usage de la cartographie qui est un outil qui permet de visualiser les problèmes fonciers, mais qui n’a pas la capacité intrinsèque de les résoudre. De surcroît, certaines ambiguïtés apparaissent ainsi maintenues dans le contexte du jeu de rôle dès lors que les demandes foncières présentées par les acteurs sont rappelées dans des termes qui évacuent la question foncière en tant que telle. Ainsi les projets présentés relèvent d'une problématique de reboisement (pour le premier), de relation de genre (pour le second), ou de développement des produits d'élevage (pour le troisième). Même si on reste dans des projets hypothétiques, il est intéressant de voir s'affirmer des registres concrets de négociations récurrents qui dépassent la seule implication individuelle des présentateurs et se situent dans un argumentaire environnementaliste ou développementaliste. L'efficacité d'un tel dispositif pour le développement des connaissances sur les modes de gestion du foncier passe par une clarification du rapport au réel et de la portée du projet. En effet, le jeu se situe entre des questions foncières abstraites et des négociations concrètes. Bien que ces dernières puissent revêtir un caractère euphémique, elles ne sont cependant pas la pâle imitation des délibérations d'un conseil rural, elles ouvrent aussi aux acteurs la possibilité de répéter, au sens d'un entraînement, les négociations réelles.

Cependant, dans une démarche d'empirisme pur et en partant du principe implicite que « moins on en connaît sur les rapports sociaux à l'œuvre dans la société concernée, mieux on peut agir en toute neutralité », se pose d'emblée une question d'éthique. En effet, une telle disposition peut être sujette à caution et productrice d'erreurs tant scientifique qu'idéologique dès lors que le contexte, traversé par des rapports sociaux très hiérarchisés au sein même des familles et des clans (nobles/castés) comme au sein des hiérarchies administratives et politiques, est nié (du moins le temps d'une simulation). De plus, dans l'acte de communication, les différents rapports statutaires se superposent dans des registres de légitimation différents (Boltanski et Thévenot, 1991). S'expriment ici les rapports domestiques, hiérarchiques et familiaux, rapports historiques entre castes ou catégories sociales, d'un côté, les relations avec les catégories technocratiques et les acteurs administratifs d'un autre côté, enfin le rapport au parti pour les acteurs politiques. Ces registres de légitimation que chaque acteur utilise se superposent et s'imbriquent étroitement. On peut supposer que les relations domestiques structurent autant les actes d'un président de conseil rural (PCR), lorsque celui-ci est issu du milieu, que sa fidélité au parti politique.

La démarche des interventions de développement participatif implique une certaine indécision entre le « laisser-faire » et l'intervention (Lavigne-Delville et al., 2000). Elle ne produit alors qu'une définition vague et imprécise des objectifs de recherche et d'action présentés, partant du principe pragmatique que les protagonistes auront la liberté de choisir et de s'exprimer. Dans la constitution du groupe de travail, les différentes modalités de choix des acteurs par les intervenants ont pu relever de ce pragmatisme. Certains acteurs ont été choisis pour leur statut d'acteur incontournable dans la gestion des terres, tels, entre autres, les Présidents des conseils ruraux. D'autres ont été choisis pour leur «représentativité » de certains groupes de la société civile (femmes) et/ou de secteurs professionnels (pêche, maraîchage, élevage, agro-industrie). Implicitement, le choix a été composé de divers arguments dont le principal était d’y voir représentées toutes les catégories sociales et politiques concernées par la gestion foncière. Pourtant, d'un autre côté, les conditions d'accès au groupe de travail ont pu être définies et présentées aux protagonistes à partir de critères d'une autre nature. Ceux-ci supposaient de la part des acteurs à la fois un intérêt à participer, une disponibilité, un engagement et un intérêt pour l'utilisation des outils à travers une démarche qui restait bénévole. Ces dispositions permettaient d'ouvrir à un public large la constitution du groupe de travail, quand bien même elles n'ont été que très peu déterminantes du fait de leur caractère générique et des contraintes de représentativité sociale qui s'imposaient dans les objectifs du projet. 
L'ensemble n'est donc pas le fruit d'une auto-constitution, ni tout à fait celui d'une désignation par le groupe de chercheurs. Le poids relatif des acteurs (privés, publics, associatifs) n'y est cependant pas stabilisé. Certains acteurs publics manquent (services forestiers, administration) et les différents statuts d'un même acteur ne sont pas encore bien connus. Les ateliers ont cependant été construits de manière à connaître un peu mieux au-delà de la simulation, " chemin faisant » et a posteriori les protagonistes, notamment en termes d'alphabétisation, de connaissances de la carte, de participation aux projets, dans leurs motivations, et leurs responsabilités, dans leurs statuts réels, dans leurs positions sociales et leurs prises de positions dans le débat sur le foncier. G. Leclerc et al., (2008) en ont d'ailleurs fait référence. De par sa configuration, le groupe de travail est cependant susceptible de devenir un comité ad hoc qui, selon l'évolution des ateliers futurs du projet, serait à même d'accompagner les autorités locales compétentes pour gérer les ressources foncières. La question de l'autonomie et du rôle réel, légitime et légal d’un tel groupe de travail se pose dans la continuité d'un tel processus en termes de représentativité de ses acteurs, de leurs attentes et de leur engagement bénévole et dans son efficacité supposée dans les négociations futures au niveau des questions foncières.

\section{Conclusion}

L'utilisation de tels outils participatifs (jeux de rôle et cartes géographiques) demeure une démarche d'expérience dans un partenariat local donné qui révèle en dernière instance une forte influence du contexte et une incertitude dans son déroulement. La non prise en compte de ce contexte social renvoie aux limites des outils et aux risques qu'ils présentent quant à l'usage inégal de l'information produite, jouant ainsi autant à l'insu du rôle des chercheurs que de celui des acteurs locaux. Dans l'expérience du jeu de rôle, par exemple, si l'implication du chercheur dans le dispositif, en tant que modérateur d'une situation fictive, est susceptible d'orienter implicitement les termes du débat, le problème du statut social réel de chacun des acteurs n'en reste pas moins posé.

En définitive, les termes de référence du rôle du groupe de travail sont essentiellement d'apparence technique, bien que le rôle de garant qui est attribué à ce groupe puisse en même temps renvoyer directement à un potentiel futur de légitimité politique alors que certaines ambiguïtés persistent autour de son autorité réelle dans la gouvernance expérimentale actuelle. Au cours d'une telle expérience, certaines questions se posent, d'une part, quant au rôle et à l'efficacité sociale du groupe de travail, d'autre part, quant à la portée du dispositif dans la dynamique des rapports sociaux et enfin, quant à la forme institutionnelle et politique qu'il représente et/ou est en droit de constituer dans le contexte légal de gouvernance foncière. En effet, le groupe de travail peut apparaître, dans un premier temps, comme un lieu en substitution à un travail de recherche pour conduire la réflexion sur les questions foncières. Dans le même temps il se présente comme un « groupe de travail ou de réflexion » accompagné par les chercheurs pour produire des connaissances sur les problèmes fonciers, les modes de négociations et pour diffuser, dans l'espace public, les termes d'une nouvelle gouvernance en matière de gestion de l'espace (prise en compte de la mobilité des éleveurs, prise en compte des plus démunis, priorité des questions de durabilité écologique). Par ailleurs la portée d'un tel dispositif donne à voir la possibilité pour les élus locaux de renforcer leur pouvoir, sans pour autant répondre davantage aux critères d'accountability. À l'inverse, elle peut donner lieu à la production de contre-pouvoir pour mettre à l'épreuve la responsabilité politique des élus, voire transmuer son rôle en une dynamique syndicale de manière à rendre publiques les revendications de certains groupes marginalisés (femmes, éleveurs, etc.). Au vrai, contrairement à la relation d'enquête du travail de recherche, le type de "forum » (Callon et al., 2001) expérimenté ici dans le cadre d'une recherche-action, n'a pas pour dessein de rendre compte de toutes les données empiriques disponibles qui informent sur le contexte, les enjeux et les pratiques quotidiennes. C'est un dispositif qui permet de favoriser l'énonciation des points de vue respectifs des différents acteurs autour d'un sujet donné. L'adoption d'une posture post 
moderne demande cependant de prendre des précautions particulières. Un tel forum expérimental qui nierait les hiérarchies et les rapports de domination qui président aux relations sociales ne pourrait autoriser en réalité de reconnaître leurs effets bien réels sur les positions et prises de positions, et, partant, sur les décisions potentielles des acteurs.

L'enjeu fondamental d'une telle démarche, comme il est recommandé dans la relation d'enquête (Bourdieu, 1994), est de « réduire « au minimum » la violence symbolique » (Sintomer, 2006; Boutinot et Diouf, 2006) qui préside aux relations inégales, et « qui accompagne la communication entre des locuteurs aux statuts sociaux disparates » (Sintomer, 2006). À défaut de considérer cet enjeu, ce type de forum et d'expérience de démocratie participative locale, pourrait bien, à l'instar de certains projets de développement ordinaires, aboutir au renforcement des capacités de ceux qui ont déjà le pouvoir de les distinguer.

\section{BiBLIOGRAPHIE}

Adger (W. N.), Brown (K.), Fairbrass (J.), Jordan (A.), Paavola (J.), Rosendo (S.), Seyfang (G.), 2003. - « Governance for sustainability: towards a 'thick' analysis of environmental decisionmaking », Environment and Planning, vol. 35, n 6, p. 1095-1110.

Agrawal (A.), 2005. - «Communautés, gouvernement intime et sujets de l'environnement à Kumaon, Inde », Anthropologie et Sociétés, vol. 29, n 1, p. 21-47.

Agrawal, (A.), Ribot, (J.-C.), 1999. - «Accountability in decentralisation: a framework with South Asian and West African Cases », Journal of Developing Areas, vol. 33, n 4, p. 473-502.

Ayeva (T.), 2003. - « Gouvernance locale et renforcement des capacités. Quelques pistes de réflexion pour un développement territorial durable des collectivités rurales ", Cahiers de recherche du CRTD, Université du Québec à Rimouski, Québec, Canada, 49 p.

Bacque (M.-H.), 2005. - « L'intraduisible notion d'empowerment vu au fil des politiques urbaines américaines », Territoires, $n^{\circ} 460$, Septembre, p. 32-35.

Bierschenk (T.), Chauveau (J.-P.), Olivier de Sardan (J.-P.), 2000. - Courtiers en développement. Les villages africains en quête de projets, Paris, Karthala, Mayence APAD, 318 p.

BLAiR (H.), 2000. - «Participation and accountability at the Periphery: Democratic local gouvernance in six countries ", World Development, vol. 28, n 1, p. 21-39.

Blondiaux (L.) et LeVÊQue (S.), 1999. - « La politique locale à l'épreuve de la démocratie. Les formes paradoxales de la démocratie participative dans le XXe arrondissement de Paris », dans NEvEU (C.), (dir.), Espace public et engagement politique. Enjeux et logiques de la citoyenneté locale, Paris, L'Harmattan, coll. «Logiques Politiques », p. 17-82.

BlondiauX (L.), 2007. - « La démocratie participative, sous conditions et malgré tout. Un plaidoyer paradoxal en faveur de l'innovation démocratique », Mouvements, vol. 50, n 2, p. 118-129.

Blundo (G.) et Olivier de SARdan (J.-P.), 2001. - «Introduction, La corruption au quotidien », Politique Africaine, $\mathrm{n}^{\circ} 83$, p. 5-7.

Blundo (G.), 1997. - «Gérer les conflits fonciers au Sénégal : le rôle de l’administration locale dans le sud-est du bassin arachidier », dans Becker (C.), Tersiguel (P.) (dir.), Développement durable au Sahel. Dakar, Paris, Karthala coll. «Sociétés, Espaces, Temps », p. 103-122.

Boltanski (L.), Thevenot (L.), 1991. - De la justification. Les économies de la grandeur, Paris, Gallimard, 483 p.

Bourdieu (P.), 1994. - Raisons pratiques, Paris, éditions du Seuil, 251 p.

Boutinot (L.), 2002. - «De la complexité de la décentralisation. Exemple de la gestion des ressources forestières au Sénégal », Bulletin de l'APAD, n² 23, p. 37-59.

Boutinot (L.) et Diouf (C N.), 2006. - «Quand certaines approches participatives engendrent des formes ambiguës de mobilisation de la société civile. Quelques exemples à propos de la gestion des ressources naturelles au Sénégal », dans Bertrand (A.), Karsenty (A.), Montagne (P.) (dir.), L'Etat et la gestion locale durable des forêts en Afrique francophone et à Madagascar, Paris, L'Harmattan, p. 192-208. 
Laurence Boutinot, Alain A. Viau, Grégoire LeclerC

Callon (M.), Lascoumes, (P.), Barthe (Y.), 2001. - Agir dans un monde incertain. Essai sur la démocratie technique, Paris, Seuil, 358 p.

Cambrézy (L.), De Maximy (R.), (dir.), 1995. - La cartographie en débat. Représenter ou convaincre, Paris, Karthala/Orstom, 200 p.

CercCop, 2003. - Les méthodes participatives dans les projets de développement ( $1^{\text {re }}$ partie), Dossier II, Lettre $\mathrm{n}^{\circ}$ 13, Besançon, [http ://wwww.cercoop.org/lettres/let14do1.htm].

Chrisman (N.), 2005. - «Communities of Scholars: Places of Leverage in the History of Automated Cartography ", Cartography and Geographic Information Science, vol. 32, n 4, p. 425-433.

Chrisman (N.), 2007. - Exploring Geographical Information Systems, 2nd Edition, John Wiley, 320 p.

CoAse (R.H.), [1937] 1988. - The firm, the market and the law, Chicago, University of Chicago Press, 226 p.

D’AQUino (P.), 2001. - « Ni planification locale, ni aménagement du territoire : pour une nouvelle approche de la planification territoriale », Géographie, É http://farm4.static.flickr.com/3366/3246749533_87c1107d11_ s.jpg conomie, Sociétés, vol. 3, n² 2, p. 279-300.

Desrosières (A.), 2000. - La politique des grands nombres. Histoire de la raison statistique, Paris, Éditions de la Découverte/Syros, 456 p.

Diop (M.-C.), 2002. - Le Sénégal contemporain, Paris, Karthala, 656 p.

Diop (M.-C.), 2004. - Gouverner le Sénégal - Entre ajustement structurel et développement durable, Paris, Karthala, 299 p.

DjiRE (M.), 2004. - Les conventions locales au Mali: une grande nébuleuse juridique et un pragmatisme en GRN, No 2, IIED, Dakar, Edimbourg, 35 p.

Duran (P.), 2004. - « Génèse de l'analyse des politiques publiques », dans Boussaguet (L.), Jacquot (S.), Ravinet (P.), Dictionnaire des politiques publiques, Paris, Presses de Science Po, 518 p.

Ervine (K.), 2007. - « The Greying of Green Governance: Power Politics and the Global Environment Facility ", Capitalism Nature Socialism, vol. 18, n 4, p. 125-142.

Fairhead (J.), Leach (M.), 2003. - Science, Society and Power : Environmental Knowledge and policy in West Africa and the Caribbean, Cambridge, $292 \mathrm{p}$.

FAiRheAd (J.), LeAch (M.), 1998. - Reframing Deforestation: Global analyses and local realities- studies in West Africa. London, Routledge, 265 p.

Froger (G.) 2007. - «Environnement et gouvernance: de la politique publique à la régulation de l'action collective » dans Hufty (A.), Freire (M.-D.), Plagnat (P.), Neumann (V.), Jeux de gouvernance: regards et réflexions sur un concept, Édition IUED-Karthala, p. 29-51.

GueYe (B.), TAll (M.), 2001. - Apprendre ensemble, IIED, Sahel, 32 p.

Granier, (L.), 2006. - Les conventions locales de gestion des ressources naturelles et de l'environnement. Légalité et cohérence en droit sénégalais, UICN, Gland, Suisse et Cambridge, Royaume-Uni, X + 44 p.

Harvey (F.), 2000. - « The social construction of Geographical Information Systems », International Journal of Geographical Information Sciences, vol. 14, n 8, p. 711-713.

Harvey (F.), Chrisman (N. R.), 1998. - « Boundary objects and the Social Construction of GIS Technology », Environment and Planning Annals, vol. 30, p. 1683-1694.

Healey, (P.), 2005. - «Place, Identity and Governance: Transforming Discourses and Practices » dans Hillier (J.), Rooksby (E.) (dir.), Habitus: A Sence of Place, Burlington, Ashgate, p. 189-218,

Hufty, (M.), 2007. - « L'objet de gouvernance », dans Hufty (A.), Freire (M. D.), Plagnat (P.), Neumann (V.), (dir.), Jeux de gouvernance : regards et réflexions sur un concept, Paris, IUED/Karthala, p. 13-28.

Ilboudo (J.-P.), 2002. - La radio rurale : outil d'enquête sociale, DRVF, FAO, Rome, Service de la vulgarisation, de l'éducation et de la communication. $122 \mathrm{p}$.

Johnson (J. T.), Pualani Louis (R.), Hadi Pamono (A.), 2006. - « Facing the Future: Encouraging Critical Cartographic Literacies In Indigenous Communities » ACME, vol. 4, n 1, p. 80-98.

LaCoste (Y.), 1982. - La géographie ça sert d'abord à faire la guerre, Paris, Maspero, 213 p.

Lascoumes (P.), Le Gales (P.), 2004. - Gouverner par les instruments, Paris, Presses de la Fondation Nationale de Sciences politiques, $372 \mathrm{p}$.

Lavigne-Delville (P.), Sellamna (N.-E.), Mathieu (M.-L.), (dir.), 2000. - Les enquêtes participatives en débats. Ambitions, pratiques et enjeux, Paris, Editions du GRET, Karthala, ICRA, 543 p. 
LECLERC (G.), 2007. - Photographies issues du portfolio de l'atelier de formation en cartographie, Sénégal Keur, Momar Sarr, n.p.

Leclerc (G.), Bah (A.), Diop Gaye (I.), Soumare, (A.), 2008. - « Mieux gérer les "compétences à problèmes" : retour d'expérience d'une démarche de modélisation d'accompagnement sur les usages des terres dans la zone du Lac de Guiers au Sénégal », Actes du Colloque International Gouvernance Locale et Gestion Décentralisée des Ressources Naturelles (CRDI/CSE), Dakar 12-13 février, (sous presse).

Milani (C. R. S.), 1999. - «Gouvernance territoriale », dans UIEP Paris, Mondialisation: Les Mots et les choses, books.google.com, La globalisation, les organisations internationales et le débat sur la gouvernance, UNESCO-IEP, Paris URL : [http://books.google.ca/books?hl=frElr=Eid=ck8Gwzgiar0CEoi=fndEpg=PA16 $9 \varepsilon d q=\% 22 \mathrm{MILANI} \% 22+\% 22 \mathrm{La}+$ globalisation, + les+organisations + internationales + et $+. . \% 22+$ Eots $=K K 7 x-$ X3qLJEsig=5o-7ydDA6ZjcuHCS6Z8212sPFVs\#PPA340,M1].

Monmonier (M.), 1993. - Comment faire mentir les carte? Du mauvais usage de la géographie, Paris, Flammarion, $235 \mathrm{p}$.

Mosse (D.), 2000. - «Autorité, genre et savoirs : réflexions théoriques sur la pratique du diagnostic participatif en milieu rural », dans Lavigne Delville (P.), Sellamna (N.-E.), Mathieu (M.-L.) (dir.), Les enquêtes participatives en débats. Ambitions, pratiques et enjeux, Paris, Editions du GRET, Karthala, ICRA, p. 359-392.

Muller (P.), 1990. - Les politiques publiques, Paris PUF, coll. «Que sais-je? » 128 p.

Rambaldi (G.), Chambers (R.), McCall (M.), Fox (J.), 2006. - «L'éthique dans la pratique pour les praticiens, les facilitateurs, les intermédiaires en technologie et les chercheurs en matière de SIGP », Participatory Learning an Action, 54, London, IIED, p. 13-19.

Roy (F.), VIAU (A. A.), 2007. - « Les instruments de gestion foncière : des bases incontournables pour la gouvernance des territoires en Amérique latine », Joint Congress of the European Regional Science Association (47th Congress) and ASRDLF (Association de Science Régionale de Langue Française, 44th Congress), Paris, 29 août-2 septembre, 14 p.

Sellamna (N.-E.), 2000. - « Du Rapid Rural Apparaisal au Participatory Learning and Action : la participation a-t-elle besoin d'un paradigme scientifique? », dans Lavigne Delville (P.), Sellamna (N.-E.), Mathieu (M.-L.) (dir.), Les enquêtes participatives en débats. Ambitions, pratiques et enjeux, Paris, Karthala/Éditions du GRET/ICRA, p. 453-496.

Sieber (R. E.), 2000. - « Conforming (to) the Opposition: The Social Construction of Geographical Information Systems in Social Movements », International Journal of Geographic Information Science, vol. 14, p.775-793.

Sintomer (Y.), 2006. - « La critique intellectuelle entre corporatisme de l'universel et espace public », dans Muller (H.-P.), Sintomer (Y.) (dir.), Pierre Bourdieu, théorie et pratique, perspectives franco-allemandes, Paris, La Découverte, coll. «Recherches », p. 207-222.

Viau (A. A.), Boutinot (L.), 2007. - « Géomatique et gouvernance territoriale : outils, connaissances et expertise », Joint Congress of the European Regional Science Association (47th Congress) and ASRDLF (Association de Science Régionale de Langue Française, 44th Congress), Paris, 29 août-2 septembre $18 \mathrm{p}$.

YODA (B.), 2004. - Montage et gestion participative des projets de développement rural : outils et méthodes d'intervention, Mémoire de $3^{\mathrm{e}}$ cycle en Agronomie, École Nationale d’Agriculture, Meknès, Maroc, 219 p.

Cet article a été reçu le 12 avril 2008 et définitivement accepté le 30 janvier 2009. 